\title{
Estudio de Utilización de Clonazepam Tabletas Basado en la Prescripción Ambulatoria en el Departamento del Atlántico durante el Primer Semestre de 2010
}

\author{
Utilization Study Based on Tablets Clonazepam Outpatient \\ Prescription in the Department of the Atlantic during the first half \\ of 2010
}

\author{
González Miranda J', Ruidíaz Rodríguez $\mathrm{K}^{2}$, Hernández Gámez $\mathrm{O}^{3}$, Borges Bonadiez D4. \\ ${ }^{1}$ Coomeva EPS (Baq, Atl, Col), ${ }^{2}$ Laboratorios INCOBRA (Baq, Atl, Col), ${ }^{3}$ Universidad del Atlántico (Baq, Atl, Col), ${ }^{4}$ Secretaria de Salud del Departamento del \\ Atlántico (Baq, Atl, Col)
}

\begin{abstract}
Resumen - Los estudios de utilización de medicamentos permiten identificar áreas específicas de intervención que permitan mejorar la calidad terapéutica e identificar patrones de prescripción, comercialización y uso de medicamentos en una población.

Objetivo: Elaborar un estudio de utilización de Clonazepam tabletas, basado en la prescripción ambulatoria en el departamento del Atlántico durante el periodo comprendido entre Enero y Julio de 2010.

Materiales y Métodos: Se efectuó un estudio descriptivo-retrospectivo con base a los recetarios oficiales de las prescripciones ambulatorias en el Atlántico. Se reportaron 12635 recetarios, de los cuales solo fueron incluidos en el estudio 9642por criterios de inclusión-exclusión. Se les realizo un muestreo estratificado de afijación proporcional de las prescripciones, obteniéndose un tamaño de muestra de 2900 prescripciones.

Resultados: La prescripción de clonazepam fue superior en mujeres $(54,57 \%)$ que en hombres $(45,06 \%)$. Los diagnósticos más frecuentes en los cuales se prescribió Clonazepam tabletas de 0,5 y $2 \mathrm{mg}$, en las mujeres fueron esquizofrenia paranoide y el trastorno de ansioso depresiva; por su parte, en los hombres los diagnósticos más frecuentes fueron el trastorno ansioso depresivo y el trastorno de ansiedad no especificado.El médico general presenta el mayor porcentaje de prescripciones (47\%), seguido de especialistas como Psiquiatras (28,44\%) y neurólogos (2,15\%).

Conclusión: Según los resultados, en el 95,74\% de las prescripciones, la prescripción de clonazepam es inadecuada, siendo en este caso el diagnóstico más frecuente el trastorno de ansiedad mixto. Solo en un $4,26 \%$ la prescripción de clonazepam fue adecuada, siendo el diagnóstico más frecuente el trastorno del sueño no especificado.

Palabras Clave: Diagnóstico, Empresa Social de Estado, Nivel de Cumplimiento, Procesos Generales, Servicio Farmacéutico (Fuente: DeCS).
\end{abstract}

Correspondencia: Jerson González Miranda. Servicio Farmacéutico. Coomeva. Barranquilla, Atlántico, Colombia. jerson_gonzalez@coomeva.com.co.

Citar: González Miranda J, Ruidiaz Rodríguez K, Hernández Gámez O, Borges Bonadiez D. Estudio de Utilización de Clonazepam Tabletas Basado en la Prescripción Ambulatoria en el Departamento del Atlántico durante el Primer Semestre de 2010. Cienc e Innovación en Salud [Internet]. 2015 Jan 1;3(1):10-15. Available from: http://publicaciones.unisimonbolivar.edu.co:82/rdigital/ojs/index.php/innovacionsalud/article /view/234/231.

Parte de este trabajo se encuentran soportado en la investigación titulada «Estudio de Utilización de Clonazepam Tabletas Basado en la Prescripción Ambulatoria en el Departamento del Atlántico durante el Primer Semestre de 2010» tesis para obtener el título de Químico Farmacéutico de la Universidad del Atlántico (Colombia).

Recibido: Oct. 12 de 2014 / Modificado: Nov. 11 de 2014 / Aceptado: Nov. 21 de 2014.
Abstract - Pharmaceutical Service is responsible for the activities, Processing and Interventions Technical, scientific and administrative character, relating to Drugs and Medical Devices.

Objective: Determine the level of fulfillment as for the existence and application of procedures for general processes (Selection, Acquisition, Receipt, Storage, Distribution and Dispensation of medicines and medical devices) in pharmaceutical services of Social Companies of State of the department.

Materials and methods: Cross-sectional study. The study population consisted of twenty-five (25) classified as ESE Hospitals, Center for Health and Local Health Units in the Department of the Atlantic; to collect information previously validated instrument was used by verification experts panel.

Results: Fulfillment of General Processes is High Degree of Complexity with $87,40 \%$, followed by Medium Degree of Complexity with $54,00 \%$ and the minor value of Low fulfillment Degree of Complexity with 40,94 $\%$, the major percentages of fulfillment for process and Degrees of Complexity were: Selection with $88,07 \%$ for High Degree of Complexity; Acquisition with $77,17 \%$, for Medium Degree of Complexity; Receipt and Storage with 90,24\% for High Degree of Complexity; Distribution with $97,25 \%$ for High Degree of Complexity and Dispensation with 85,32\% for High Degree of Complexity.

Conclusions: General For all processes, the compliance was lower subvariable Control Actions during the process, which shows that the Pharmaceutical Companies Social Services of the State, have not properly defined and implemented indicators to measure the performance of the processes under investigation.

Keywords: Diagnosis, Fulfillment, Pharmaceutical Service, Procedure (Source: MeSH, NLM).

\section{INTRODUCCIÓN}

Desde las más antiguas civilizaciones el hombre ha utilizado productos de origen vegetal, mineral, animal o en los últimos tiempos sintéticos como forma de alcanzar mejoría en distintas enfermedades (1)para logra de esta manera un mejoramiento sustancial de su modus vivendi.

En este sentido, la evolución de la ciencia farmacéutica ha contribuido al descubrimiento, desarrollo y elaboración de medicamentos destinados a 
tratar la gran mayoría de las enfermedades que afectan al hombre, siendo así los medicamentos una herramienta vital para la mejoría de la calidad de vida de millones de personas alrededor del mundo. Desafortunadamente, tal vez por desconocimiento tanto de profesionales de la salud como de las personas del común, muchas veces los medicamentos se usan de forma inapropiada, pudiéndose convertir en una amenaza para la salud tanto individual como colectiva, llegando a producir efectos indeseados, que en algunas ocasiones pueden resultar mortales.

Entre las consecuencias derivadas del mal uso de los medicamentos podemos mencionar, entre otros, la pérdida de la efectividad (observada muy a menudo en antibióticos), discapacidad e incluso la muerte, llegando en muchas ocasiones a gastos elevados en terapias alternativas. La Organización Mundial de la Salud (OMS) señala que "en el mundo, más de la mitad de todos los medicamentos que se prescriben, se dispensan o se venden indebidamente, y la mitad de todas las personas no los toman correctamente", lo que revela que el uso racional de medicamentos (URM) se convierte en una estrategia de grandes y positivas consecuencias, y permite enfrentar la enfermedad de forma efectiva y eficiente (2).

Para llevar a un uso racional de los medicamentos la OMS ha reconocido la necesidad de establecer una política nacional de medicamentos y la importancia de una estrategia asociada de investigación que incluya estudios del uso de los medicamentos. Considera además, que los estudios de utilización de medicamentos son un tema prioritario y los define como aquellos que tienen por objeto de análisis la comercialización, distribución, prescripción y uso de los medicamentos en una sociedad, con acento especial sobre las consecuencias médicas, sociales y económicas resultantes (3)

Existen pocas dudas que la magnitud de la práctica sub-óptima de prescripciones (tanto por exceso como por defecto) sobrepasa el costo mínimo de los medicamentos. Hay suficientes evidencias que señalan que a pesar de la existencia de guías de manejo, existe una gran variabilidad de la prescripción, la cual va más allá de lo esperable por la variedad en la presentación clínica de los pacientes. Una de las formas de prescripción inadecuada se manifiesta cuando no se prescriben medicamentos para la patología en la cual está claramente demostrada su utilidad (4). Por ejemplo el uso de los psicofármacos ha adquirido una importancia relevante, en especial por el aumento constante y creciente de las patologías que requieren su empleo y por la disponibilidad actual de recursos farmacológicos de menor toxicidad. La utilización en forma racional se basa en un correcto diagnóstico, el cual lleva implícito un pronóstico y permite la elección del o los recursos terapéuticos más adecuados. Es cuestionable el hecho de que la mayoría de los psicofármacos sean prescriptos por médicos generales (2-4), lo que permite inferir que representan indicaciones para episodios agudos, o la simple repetición de la receta que anteriormente fuera hecha o no por un especialista. Esto no responde a un tratamiento racional con el seguimiento, evaluación y tiempo de terapéutica adecuado para el paciente (5).

El Clonazepam tiene un perfil muy eficaz como ansiolítico en el tratamiento de trastornos, pero el consumo elevado de Clonazepam constituye una de las preocupaciones más relevantes en las instituciones de salud, por las implicaciones serias en los pacientes que conlleva la utilización crónica de los mismos (6), pueden desarrollarse mecanismos de tolerancia y dependencia física, así como efectos secundarios menos graves como somnolencia y falta de concentración diurna, los cuales pudieran provocar complicaciones en las actividades cotidianas de los pacientes, además, pueden presentarse algunos problemas de interacciones medicamentosas (7). Es por esto que el uso crónico de este medicamento se convierte en una problemática de salud pública.

Es de particular interés analizar el comportamiento de utilización de medicamentos de uso controlado y monopolio del Estado, ya que según las consideraciones tenidas en cuenta en la Resolución 1478 de 2006, "estos medicamentos crean dependencia" y "su uso inadecuado conlleva al manejo ilícito de los mismos"(8).Dentro de los medicamentos de control especial establecidos en esta resolución encontramos las benzodiacepinas, utilizadas como hipnóticos y sedantes para el tratamiento del insomnio pertinaz patológico, ansiedad traumática generalizada, convulsiones y crisis de pánico incapacitantes, en este caso como opción de segunda línea $(9,10)$.

La Secretaría de Salud Departamental del Atlántico ha observado que el consumo de Clonazepam en el año 2009 fue superior al consumo reportado para el año 2008, tendencia que de continuar en el año 2010 sería motivo de preocupación. Es por esto que surgen diversos interrogantes como: ¿qué influencia puede tener la forma como se prescribe el Clonazepam con el aumento del consumo del mismo?, ¿qué tan adecuada es la prescripción de Clonazepam de acuerdo a guías de tratamiento reconocidas a nivel nacional e 
internacional? y ¿̇e ajusta la prescripción ambulatoria de Clonazepam en el departamento del Atlántico a los requisitos legales contenidos en la Resolución 1478 de 2006? La presente investigación nació de la necesidad de realizar un estudio de prescripción - indicación en el departamento del Atlántico con la finalidad de determinar la adecuación de la prescripción a guías de tratamiento de psicofármacos reconocidas en el país.

\section{MATERIALES Y MÉTOdOS}

La presente investigación se realizó en el departamento del Atlántico está situado al norte del territorio nacional. Se revisaron las prescripciones que contengan clonazepam en la forma farmacéutica de tabletas, en las concentraciones de $0,5 \mathrm{mg}$ y $2 \mathrm{mg}$ que se encuentren en los recetarios oficiales reportados a la Secretaría de Salud Departamental del Atlántico durante el periodo comprendido entre enero y agosto del año 2010.

Se establecieron como criterios de inclusión aquellas prescripciones que tengan consignados los datos referentes a la edad del paciente, sexo del paciente, medicamento prescrito (Clonazepam tabletas $0,5 \mathrm{mg}$ y 2 $\mathrm{mg})$, dosis, diagnostico, pauta de la prescripción y especialidad del prescriptor. Fueron excluidas todas aquellas prescripciones en las cuales el diagnóstico no se encuentre o sea ilegible y aquellas que correspondan a consolidados de consumo realizados por los servicios de las diferentes clínicas y hospitales.

El marco muestral se constituyó por el listado de fórmulas con diagnostico completo. Es importante destacar que este listado fue verificado y actualizado de acuerdo con las recomendaciones de la Secretaria de Salud Departamento del Atlántico, esto con la finalidad de asegurar que el directorio de fórmulas estuviese completo y actualizado.

El tipo de muestreo fue de tipo probabilístico estratificado proporcional. Se consideró tamaño de la muestra será un porcentaje del total de unidades del directorio.Igualmente, se consideró el caso más general de calcular el tamaño de muestra para estimar la proporción mediante muestreo aleatorio simple, ya que en la práctica no se tienen datos de la variabilidad de cada estrato.

Luego de introducir la información en la base de datos, se procedió a realizar los análisis estadísticos, utilizando medidas de tendencia central como la media, mediana y frecuencia para el análisis del consumo de los medicamentos, distribución etaria de las prescripciones. Se utilizó una prueba de acierto y desacierto para determinar si las prescripciones se adecuaron a las guías de tratamiento para lo cual, previamente se debió hacer una evaluación de las prescripciones tomando como referencia guías de tratamiento reconocidas a nivel nacional e internacional. Finalmente se realizó una prueba de acierto y desacierto para verificar que las prescripciones cumplieron con los requisitos establecidos en la en la Resolución 1478 de 2006. Los análisis estadísticos se realizaron con el paquete Estadístico cran R-proyect.

\section{RESULTADOS}

En el departamento del Atlántico, en el periodo comprendido de Enero a Julio del 2010, se reportaron 12635 recetarios oficiales en los cuales se prescribió Clonazepam tabletas de 0,5 y $2 \mathrm{mg}$, de los cuales solo fueron incluidos en el estudio 9642 recetarios debido a que cumplían con los criterios de inclusión y exclusión. A estos recetarios oficiales, se realizó un muestreo estratificado de afijación proporcional de las prescripciones, obteniéndose un tamaño de muestra de 2900 prescripciones.

Al analizar los datos de la muestra, se determinó cuáles eran los diagnósticos más frecuentes en los cuales se prescribió Clonazepam tabletas de 0,5 y $2 \mathrm{mg}$, de los cuales se decidió analizar los diagnósticos que correspondieran al $80 \%$ de la frecuencia acumulada, obteniéndose un total de 2419 prescripciones a analizar.

La mayor frecuencia de prescripción de Clonazepam se encuentra en el género femenino con un $54,57 \%$ sobre el masculino con $45,06 \%$ con una diferencia porcentual de $10 \%$. También se observa que el $0,37 \%$ no reporta el género del paciente al cual se prescribe el medicamento de control. El grupo etáreo para el cual se prescribió con mayor frecuencia Clonazepam fue de 41 años a 51 años, la menor cantidad de tabletas prescritas de Clonazepam se encontraron para aquellas edades mayores a 90 años; la edad promedio de la población de estudio fue de 51 a 60 años.La edad $54 \pm 4$ años. Tanto para los hombres y mujeres, el grupo etáreo para el cual se prescribió con mayor frecuencia el Clonazepam fue de 41 años a 51 años.

Se observó un predominio en la prescripción por parte del médico general de un $47 \%$ sobre otras especialidades y el $21,25 \%$ de las prescripciones no reporta la especialidad del prescriptor. Así mismo, se observa que el $99,26 \%$ de las prescripciones no reporta el régimen al que pertenece el paciente. 


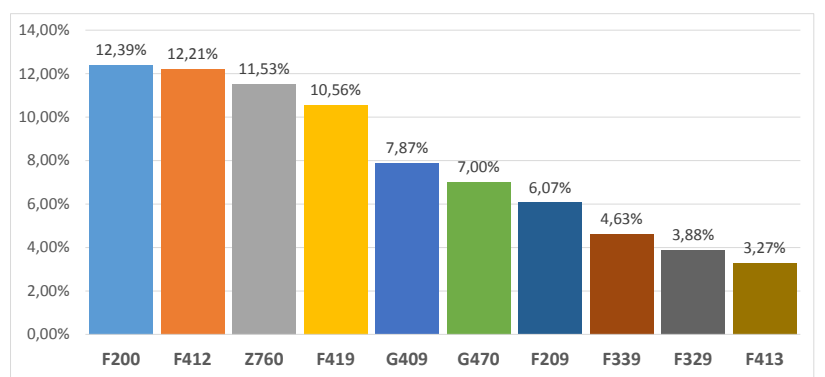

Ilustración 1 Frecuencia de indicaciones en las cuales se prescribió Clonazepam. Fuentes: Datos del Estudio.

La mayor frecuencia de prescripción de Clonazepam en forma genérica con un $91,51 \%$ con respecto al consumo de medicamentos comerciales con un porcentaje de $8,39 \%$. Mostrándose un alto índice de prescripción de medicamentos genéricos. Se observa que la mayor prescripción de Clonazepam estuvo asociada a la esquizofrenia paranoide (F200) con un porcentaje de $12 \%$, seguido por el Trastorno Ansioso Depresivo (F412), Consulta para repetición de receta (Z760) , y en menores porcentajes de frecuencias se encontró para las indicaciones de Trastorno de ansiedad, no Especificado (F419), Epilepsia, tipo no especificado (G409) 10\%, Insomnio (G470) 7\%, Esquizofrenia, no especificada (F209) 7\%, Trastorno depresivo recurrente no especificado (F339), y el 27\% correspondió para otros diagnósticos.

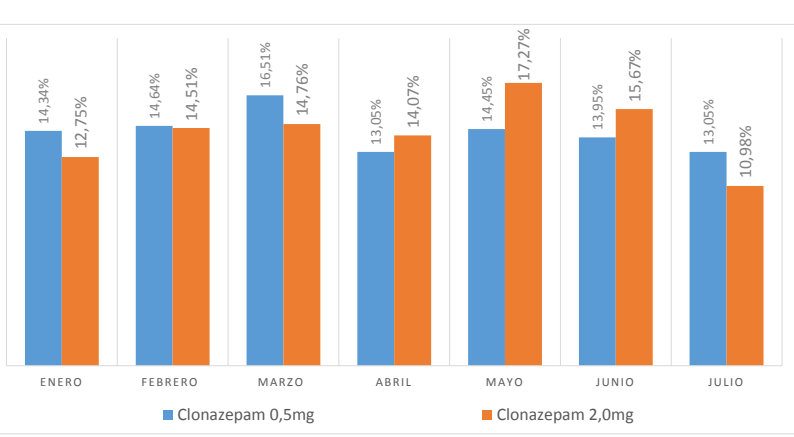

Ilustración 2 Número de prescripciones por mes de Clonazepam2,0mg y $0,5 \mathrm{mg}$ en el departamento del Atlántico

Se observa que durante el periodo de enero a julio del 2010, el mes durante el cual se reportó más prescripciones de Clonazepam0,5mgfue el mes de marzo con 592 (16,51\%) prescripciones reportadas, también se observa que no hubo una mayor diferencia entre las frecuencias de los meses reportados. Se observa que durante el periodo de enero a julio del 2010, el mes que mayor reporte hizo del consumo de Clonazepam $2.0 \mathrm{mg}$ fue el mes de mayo con un $17,27 \%$, también se observa que no hay una diferencia significativa en la frecuencia de los reportes de prescripciones por mes. Se observa la evolución mensual del prescripción de Clonazepam 2,0mg, el mes que más reportes hizo durante el periodo de enero a julio del 2010 fue el mes de mayo con un $17,27 \%$.

Tabla 1 Cumplimiento de los requisitos legales para la prescripción de Clonazepam.

\begin{tabular}{lcc}
\hline \multicolumn{1}{c}{ Ítem } & $\begin{array}{c}\text { Prescripciones que } \\
\text { cumplen }\end{array}$ & $\begin{array}{c}\text { Prescripciones que NO } \\
\text { cumplen }\end{array}$ \\
\hline Régimen del SGSS & $18[0,70 \%]$ & $2401[99,30 \%]$ \\
Tipo de Institución & $622[25,70 \%]$ & $1798[74,30 \%]$ \\
Edad del paciente & $1777[73,50 \%]$ & $642[26,50 \%]$ \\
Especialidad del & $1905[78,80 \%]$ & $514[21,20 \%]$ \\
prescriptor & $2410[99,60 \%]$ & $9[0,40 \%]$ \\
Genero del paciente & & \\
\hline Fuente: Datos del Estudio & &
\end{tabular}

Fuente: Datos del Estudio

En cuanto al cuanto cumplimiento de los requisitos legales exigidos para la prescripción de medicamentos de control especial según lo establecido en la Resolución 1478 de 2006. Se observó que el ítem que menos diligencian los médicos son el régimen SGSS al cual está afiliado el paciente con un porcentaje 99,3\% prescripciones, Tipo de institución de afiliación con un porcentaje del $74.3 \%$ prescripciones.

En cuanto a la frecuencia de 20 a 40 días es la mayor prevalencia de tiempo en días de prescripción de Clonazepam con un porcentaje del $90 \%$, para aquellas prescripciones que contenían periodo menor de 20 días tuvo un porcentaje menor al $4 \%$.

\section{DISCUSIÓN}

Se observa que la prevalencia sobre la prescripción de Clonazepam, predominó en el sexo femenino con un $54,57 \%$ sobre el masculino con $45,06 \%$. Estos resultados coinciden con los reportados en estudios realizados en Cuba $(11,12)$, México $(6)$, Chile $(13,14)$ y España (14) donde también se evidenciaba una mayor prevalencia de prescripciones en mujeres que en hombres.

Se puede observar que en los resultados la mayor parte de las prescripciones fueron realizadas por el médico general con un $47 \%$, seguido de Psiquiatras con un porcentaje del 28,44\% y neurólogos con el 2,15\%. Estos resultados coinciden con el estudio realizado por Cifuentes M. y Cols. (15) donde se observaba una similar distribución entre las especialidades de prescriptores más frecuente y con el estudio hecho por Milazzo J. y Cols(16) donde observaron que la mayor parte de prescripciones de benzodiacepinas eran realizadas por médicos generales.

Se observa que los diagnóstico más frecuentes asociados a la indicación de Clonazepam a nivel ambulatorio fueron Esquizofrenia paranoide, trastorno 
ansioso depresivo, epilepsia e insomnio, confirmando los resultados hallados en estudios acerca del uso de benzodiacepinas $(6,11,14)$ en los cuales el mayor porcentaje de prescripciones se daba en estas mismas patologías. Cabe destacar que en $12 \%$ el diagnóstico que aparecía en los recetarios era consulta para repetición de receta (Z760), el cual no constituye un diagnóstico de estudio a menos que esté asociado a otro diagnóstico, también destacamos que en un 0,51\% el diagnóstico fue hipertensión arterial, y no existe evidencia de que este fármaco aporte algún beneficio a esta indicación.

Según lo observado en el estudio se encontró que el régimen del SGSS es el requisito de la prescripción que menos tienden a llenar los prescriptores. Durante este estudio se encontró que $99,3 \%$ de las prescripciones no reportaron el régimen al cual pertenece el paciente, también se pudo determinar que el tipo de institución al que pertenece el paciente es el segundo requisito que menos tienden a llenar los prescriptores con un porcentaje de $74,3 \%$. Vemos pues que los porcentajes de incumplimiento de estos requisitos son altos y que sin embargo, los medicamentos son dispensados sin tener en cuenta el no cumplimiento de lo establecido en la resolución 1478 del 2006.

Según las guías de tratamiento consultadas en el Centro Atención de Rehabilitación Integral E.S.E. CARI, el tratamiento ambulatorio de la esquizofrenia paranoide incluye el uso de benzodiacepinas solo en el caso de que el paciente presente insomnio o ansiedad y en tal caso, el medicamento de elección es el lorazepan, por tal razón en el tratamiento de la esquizofrenia, el uso del Clonazepam sólo es justificado como coadyuvante a sintomatologías secundarias a la esquizofrenia y en los casos de urgencia si el paciente presenta agitación para lo cual se utiliza Clonazepam 2 mg cada 6 a 8 horas (17).

En cuanto a los trastornos de ansiedad, según la Asociación Colombiana de Neurología, el uso de benzodiacepinas está indicado en casos urgencia o cuando existe riesgo para el paciente por inminente pérdida laboral o académica, utilizándose dosis que van desde $0.5 \mathrm{mg} /$ día hasta los $6 \mathrm{mg} /$ día. A nivel internacional se recomienda el uso de benzodiacepinas de acción corta y por un periodo de tiempo que no exceda las 4 semanas de tratamiento, y cuando sea crucial el control rápido de los síntomas o mientras se espera a la respuesta del tratamiento con antidepresivos o TCC, además se recomienda que durante el tratamiento de la ansiedad conviene asociar Benzodiacepinas (de acción inicial rápida) con antidepresivos (acción diferida), por lo cual el Clonazepam al ser una benzodiacepina de acción larga no estaría recomendada, por lo general se recomienda el uso de Alprazolam y Bromazepam(18).

Dado que la fuente de información de este estudio de prescripción-indicación de Clonazepam se basó en la información procedente de los recetarios oficiales reportados a la secretaria de salud del departamento del Atlántico, por lo cual es una limitante del estudio ya que estos no contemplan la información clínica global del paciente. Durante este estudio se hizo la comparación de la adecuación de la prescripción basadas en guías reconocidas.

Se observa que los diagnósticos inadecuados para la prescripción de Clonazepam fueron los siguientes: otros trastornos de ansiedad mixto (F413), Esquizofrenia Paranoide (F200), Trastorno ansioso depresivo (F412), Trastorno de ansiedad no especificada (F419), Epilepsia tipo no especificada (G409), Insomnio (G470), Esquizofrenia no especificada (F209), Trastorno depresivo recurrente no especificado (F339), Trastorno depresivo no especificado(F329),episodio depresivo no especificado (F413), Hipertensión esencial primaria (I10X), y otros diagnósticos

Durante el estudio se analizaron un total de 2419 prescripciones de las cuales $103(4,26 \%)$ prescripciones resultaron adecuadas y $2316(95,74 \%)$ prescripciones fueron inadecuadas en relación a la indicación del medicamento.

\section{AGRADECIMIENTOS}

Al profesor Henry González Torres, Bio, Esp. Estad. Apl., MSc (Cand) Bio - GenPop, Coordinador de Investigaciones del Programa de Medicina de la Universidad Simón Bolívar por los aportes y correcciones de este artículo.

\section{REFERENCIAS BIBLIOGRÁFICAS}

1. Guillen DG, Albarracin A, Arquiola E, Erill S, Montiel L, Peset JL, et al. Historia del Medicamento. Aurora, USA: Boehringerlngelheim; 1985. 99 p.

2. Marchand B, Fuentes C, Mairena F, Narváez L. Uso irracional de medicamentos. Una práctica que limita beneficios, aumenta riesgos y malgasta recursos. Boletín AIS-COIME. 2009;41:1-4.

3. Committee WHOE, others. The use of essential drugs. World Health Organ Tech Rep Ser. 2000;895:1.

4. Pastó-Cardona L, Masuet-Aumatell C, Bara-Oliván $B$, Castro-Cels I, Clopés-Estela A, Pàez-Vives $F$, et al. Estudio de incidencia de los errores de medicación en los procesos de utilización del medicamento: prescripción, transcripción, validación, preparación, dispensación y administración en el ámbito hospitalario. FarmHosp. Elsevier; 2009;33(5):257-68.

5. Crismon ML. Psychotropic drugs in the elderly: 
principles of use. Am Pharm. 1990;NS30(12):57-63.

6. Del Carmen Milán R, Alonso MJ, Vigna M, Flores ME, Romano S. Utilización de tabletas de clonazepam en la consulta externa de un hospital general. RevMex Ciencias Farm. Asociaci\{ó\}n Farmac\{é\}utica Mexicana, AC; 2009;40(2):19-25.

7. Licata SC, Rowlett JK. Abuse and dependence liability of benzodiazepine-type drugs: GABAA receptor modulation and beyond. PharmacologyBiochemistry and Behavior. 2008. p. 74-89.

8. Organización Mundial de la Salud OPDLS. Promoción del uso racional de medicamentos: componentes centrales. Perspectivas políticas sobre medicamentos de la OMS. 2002.

9. World Healt Organization. The selection of essential drugs: report of the WHO Expert Committee. WHO; 1977.

10. Ministerio de la Protección Social. Por la cual se expiden normas para el control, seguimiento y vigilancia de la importación, exportación, procesamiento. República de Colombia; 2006.

11. Diez Córdova JP, Debesa García F, Sosa Lorenzo IA, García Roche RG, Bonet Gorbea MH, Fernández Larrea N. Estado de la prescripción de medicamentos en la atención primaria de salud de Ciudad de La Habana, 1997. Rev Cuba Med Gen Integr. 1999, Editorial Ciencias M\{é\}dicas; 1999;15(2):140-50.

12. Cifuentes $M$, Suárez R, Rey R, Ebner $D$, Hernández $C$. Prescripción de Benzodiazepinas despachadas en farmacias privadas de la jurisdicción del servicio de Salud Concepción Arauco. Unidad Salud MentServ Salud Concepción Arauco Chile. 1996;

13. Silva Herrera L, Vergara Fabián E, YeraAlós I, Freijoso E. Utilización de benzodiazepinas en la Atención Primaria de Salud. Rev Cuba Med Gen Integr. 1999, Editorial Ciencias M\{é\}dicas; 2002;18(3):187-90.

14. Galleguillos T, Risco L, Garay JL, González M, Vogel $M$, others. Tendencia del uso de benzodiazepinas en una muestra de consultantes en atención primaria. RevMedChil. SciELO Chile; 2003;131(5):535-40.

15. Milazzo JD, Perna AM. Análisis descriptivo de las prescripciones de psicofármacos en una población de la ciudad de Mar del Plata (Argentina). Període Febrero 2000 a Febrero 2001. Acta FarmBonaer. 2004;23(2):220-5.

16. Valencia M, Rascón ML. Abordaje psicoterapéutico de la esquizofrenia en pacientes agudos y crónicos. RevlbroamericanaPsicol. 1998;8(4):18-33.

17. Escobar F. Diagnóstico y tratamiento de los trastornos de ansiedad. Guía de Manejo de Neurología [Internet]. Bogota (Col): Asociación
Colombiana de Neurología; 2010. p. 177-89. Availablefrom: ww. acnweb. Org

18. Mayor LC. Tratamiento médico de la epilepsia. Guía Neurológica. Santa Fe de Bogotá: Asociación Colombiana de Neurología. 1999. 1-8 p. 